\title{
Effects of Oral Antibiotics on Stool Flora and Overall Sensitivity Patterns in an Intensive Care Nursery
}

\author{
LAWRENCE GRYLACK, ${ }^{(19)}$ DONALD NEUGEBAUER, AND JOHN W. SCANLON \\ Divisions of Neonatology and Bacteriology, Columbia Hospital for Women [L.G.] and Department of Pediatrics, \\ Georgetown University School of Medicine, Washington, D.C.
}

\section{Summary}

The effects of orally administered gentamicin and colistin on stool bacterial flora and overall antibiotic sensitivity patterns were evaluated in 100 newborns at risk for neonatal necrotizing enterocolitis. Gentamicin ( $2.5 \mathrm{mg} / \mathrm{kg} \mathrm{q6h)}$ and colistin $(1 \mathrm{mg} / \mathrm{kg} \mathrm{q6h})$ were administered to randomly selected groups of $\mathbf{5 0}$ newborns for 3 wk after birth during an 11-month study period. Stools were collected on days 1,11 , and 21 and cultures were grown under aerobic conditions on three different media. Staph. epidermidis was the most common predominant organism in both antibiotic groups, whereas $E$. coli and Klebsiella were the most common Gramnegative bacteria isolated. Seventeen \% of these Gram-negative species were resistant to colistin and $9 \%$ to gentamicin, with a gradual increase occurring during the 3-wk period. On the basis of 980 positive cultures from all sites in babies in the nursery during the 11-month study, $E$. coli sensitivity to kanamycin and gentamicin ranged between $92 \%$ and $100 \%$ except for one month midway through the study when sensitivity to kanamycin was at $80 \%$ and then returned to the $92-100 \%$ range. Klebsiella sensitivity to both aminoglycosides remained greater than $95 \%$ throughout. The incidence of neonatal sepsis remained consistent at seven to nine per 1000 live births during the study. One baby of 50 in the gentamicin group developed necrotizing enterocolitis at 5 wk of age; $0 / 50$ in the colistin group had necrotizing enterocolitis (not significant).

\section{Speculation}

The results suggest that the use of oral antibiotics in a limited segment of the newborn nursery population may not have significantly adverse effects on the overall incidence of bacterial resistance to antibiotics and incidence of sepsis.

We and others have previously reported a significant reduction in neonatal necrotizing enterocolitis (NEC) associated with administering oral gentamicin when compared with placebo $(6,12)$. Because of published concerns about the possible emergence of antibiotic resistant organisms, with subsequent nosocomial infection following such prophylaxis $(11,15)$, this current study was undertaken. Oral gentamicin (Garamycin, Schering) and colistin (Coly-mycin M, Warner-Chilcott) were evaluated for their effects on stool bacterial flora as well as the impact of such prophylaxis on overall antibiotic sensitivity patterns in our Intensive Care Nursery (ICN).

\section{MATERIALS AND METHODS}

As previously reported (12), newborns with birthweights of 1500 $\mathrm{g}$ or less, all newborns with gestations less than $37 \mathrm{wk}$ and with documented histories of fetal distress, perinatal asphyxia or hypotension, and all newborns with umbilical catheters were consid- ered at risk for NEC. One hundred consecutively born NEC-risk babies who delivered between November 1978 and October 1979 were enrolled. This group constituted $14 \%$ of all ICN admissions during that time period. Their birthweight and gestational age data are shown in Table 1.

Placement into gentamicin or colistin groups was determined by random assignment through blind card draw. The hospital pharmacy acted as referee for treatment group assignment.

Gentamicin was given by mouth in a dose of $2.5 \mathrm{mg} / \mathrm{kg}$ at a concentration of $2.5 \mathrm{mg} / \mathrm{ml}$ of sterile water every $6 \mathrm{~h}$. Colistin was given p.o. in a dose of $1 \mathrm{mg} / \mathrm{kg}$ at a concentration of $1 \mathrm{mg} / \mathrm{ml}$ of water every $6 \mathrm{~h}$. The treatment period began within $12 \mathrm{~h}$ after birth and continued for 3 wk.

Oral feeding was begun after resolution of any acute illness and at least $24 \mathrm{~h}$ after removal of any umbilical catheter. Fresh breast milk, when available, or standard formula was used. Feedings were administered by nipple or intragastric, intraduodenal or intrajejeunal routes. The initial feeding volume was $5 \mathrm{ml} / \mathrm{kg}$ or less in the premature infants. All umbilical artery catheters had their tips in the thoracic aorta at the level of 9th to 11th thoracic vertebrae.

Stools from patients in both groups were collected on postnatal days 1,11 and 21. Cultures were grown under aerobic conditions on 5\% sheep blood agar, MacConkey's and desoxycholate agar media. Predominant organisms were identified and recorded in a semiquantitative fashion. In vitro sensitivities to gentamicin and colistin of the predominant organisms were assessed by the modified Kirby-Bauer disc technique (17).

A surveillance of bacterial cultures from all intensive care nursery sources and their sensitivity patterns to aminoglycosides of recovered Eschericia coli and Klebsiella were recorded on a continuous basis and analyzed by computer program (Greenhailgh Assocs., Philadelphia, PA).

A definitive diagnosis of NEC was made on the basis of standard published criteria (13). If a baby developed either NEC or definite enteropathic signs suspicious of NEC, then feedings were stopped, gastric decompression performed, parenteral antibiotics started and oral antibiotic continued.

\section{RESULTS}

Table 1 compares study groups by diagnostic criteria, Apgar scores, primary respiratory diagnosis and the presence of hypotension. The number of babies who received umbilical catheters, parenteral antibiotics or mechanical ventilation during the first 3 wk of life were also compared. There were no significant $(P<$ $0.05)$ differences between groups for any diagnostic or therapeutic categories. Parenteral antibiotic usage varied from 2-10 days. The initial combination of drugs used for treatment of suspected intrauterine sepsis was penicillin and kanamycin. This usage was identical in both groups.

Stool flora in study patients. A summary of stool analysis for 
predominant organisms in both study groups during the 3-wk period is provided in Table 2. Forty-two percent of all stools were sterile using aerobic culture conditions. This ratio decreased as postnatal age increased. Bacterial growth of any species was more common for the colistin group in the first stool. This trend reversed in favor of the gentamicin group in stool \#3. Staph. epidermidis was the most common predominant organism in both antibiotic groups during the period of stool collection. Gram-negative bacteria increased gradually as predominant organisms during the 3wk period. The incidence of resistance to colistin exceeded resistance to gentamicin in both antibiotic groups during all stool collections, but not at a significant level $(P<0.05)$. The majority of resistant organisms were $S$. epidermidis and Group D Streptococcus. Thirty-six \% of all organisms identified in the stools of the gentamicin treatment group were resistant to gentamicin, compared to $20 \%$ in the colistin group $(P<0.05)$. Resistance to colistin of predominant organisms in the colistin treatment group was $60 \%$, compared to $58 \%$ in the gentamicin treatment group (NS). The \% of resistant organisms in the stools of either group did not increase from day 1 to day 21 of the study period. In the gramnegative group, a total of $23 \mathrm{E}$. coli isolates and 23 Klebsiella isolates were identified and evaluated for sensitivity. Sixty-five \% were in the colistin prophylaxis group. For each of these bacterial species, four of $23(17 \%)$ were resistant to colistin and two of 23 (9\%) to gentamicin. The progression of the changes is demonstrated in Figure 1.

Overall nursery colonization and antibiotic sensitivity surveillance. During the 11-month study period, E. coli sensitivity to kanamycin and gentamicin in the nursery ranged between $92 \%$ and $100 \%$

Table 1. Comparison of study groups

\begin{tabular}{lcc}
\hline \multicolumn{1}{c}{ Characteristic } & $\begin{array}{c}\text { Gentamicin } \\
(N=50)\end{array}$ & $\begin{array}{c}\text { Colistin } \\
(N=50)\end{array}$ \\
\hline Birthweight (g) & $1674+696^{1}$ & $1858 \pm 782^{1}$ \\
Gestational age (wk) & $32.8 \pm 3.5^{1}$ & $33.0 \pm 4.0^{1}$ \\
Apgar scores $\leq 6$ & & \\
$\quad$ I min & 30 & 36 \\
$\quad 5$ min & 12 & 15 \\
Primary respiratory Dx & 18 & 17 \\
$\quad$ Respiratory distress syndrome & 10 & 11 \\
Chronic pulmonary insuffi- & & \\
ciency of prematurity & 17 & 14 \\
Hypotension & 24 & 33 \\
Umbilical artery catheters & 3 & 1 \\
Umbilical venous catheters & 21 & 17 \\
Systemic antibiotics & 24 & 25 \\
Mechanical ventilation & &
\end{tabular}

\footnotetext{
${ }^{1}$ Mean \pm S.D.
}

except for one month midway through the study. At that time sensitivity to kanamycin declined to $81 \%$ and then returned to the $92-100 \%$ range in subsequent months. Gentamicin sensitivity did not change. Klebsiella sensitivity to kanamycin and gentamicin remained greater than $95 \%$ during the entire study period. The data base analyzed included all positive cultures from all sites from all patients in the ICN. There were approximately 980 positive cultures obtained during the 11 -month study period. The incidence of diagnosed neonatal sepsis during this time ranged between seven to nine per 1000 live births. Fourteen \% of all intensive care nursery patients received parenteral antibiotics. There was no statistically significant difference $(P<0.05)$ in infection rates between the two groups. There was one baby with $\mathrm{NEC}$ in the gentamicin group, diagnosed 2 wk after antibiotic prophylaxis had been stopped. There was no cases of NEC in the colistin group.

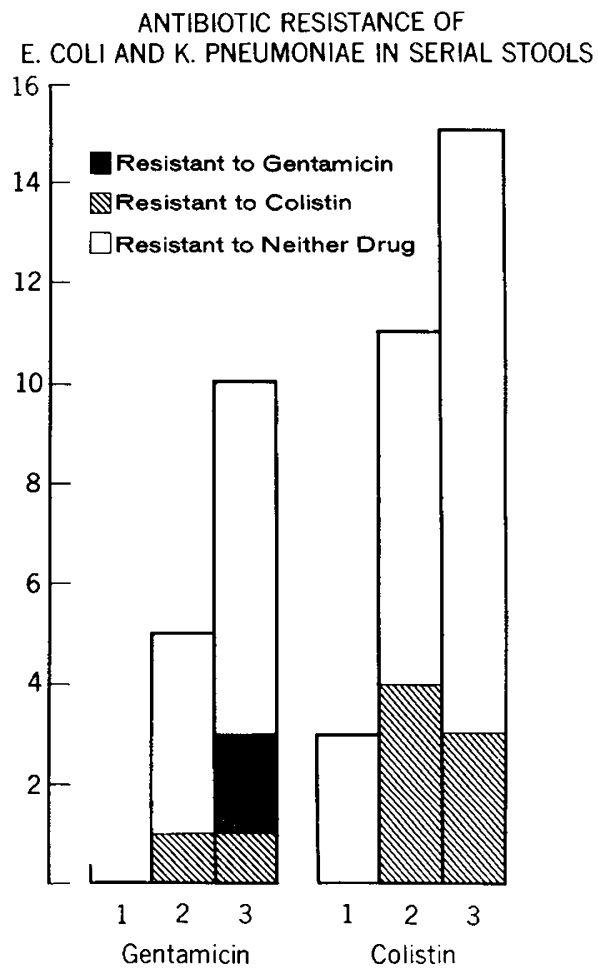

Fig. 1. Antibiotic resistance of Escherichia coli and Klebsiella pneumoniae in serial stools. The vertical axis represents the number of stools in which $E$. coli and $K$. Pneumoniae were identified as the predominant organisms. The horizontal axis represents the sequence of serial stools collected from patients in each antibiotic prophylaxis group.

Table 2. Stool flora analysis during oral antibiotic administration

\begin{tabular}{|c|c|c|c|c|c|c|c|c|c|c|c|c|c|c|c|c|c|c|}
\hline \multirow{3}{*}{$\begin{array}{c}\text { Predominant } \\
\text { organism }\end{array}$} & \multicolumn{9}{|c|}{ Gentamicin } & \multicolumn{9}{|c|}{ Colistin } \\
\hline & \multicolumn{3}{|c|}{ Stool \#1 } & \multicolumn{3}{|c|}{ Stool \#2 } & \multicolumn{3}{|c|}{ Stool \#3 } & \multicolumn{3}{|c|}{ Stool \#1 } & \multicolumn{3}{|c|}{ Stool \#2 } & \multicolumn{3}{|c|}{ Stool \#3 } \\
\hline & $\mathrm{n}^{1}$ & $\mathrm{rG}^{2}$ & $\mathrm{rC}^{3}$ & $\mathrm{n}^{1}$ & $\mathrm{rG}^{2}$ & $\mathrm{rC}^{3}$ & $\mathrm{n}^{1}$ & $\mathrm{rG}^{2}$ & $\mathrm{rC}^{3}$ & $\mathbf{n}^{1}$ & $\mathrm{rG}^{2}$ & $\mathrm{rC}^{3}$ & $\mathbf{n}^{1}$ & $\mathrm{rG}^{2}$ & $\mathrm{rC}^{3}$ & $\mathrm{n}^{1}$ & $\mathrm{rG}^{2}$ & $\mathrm{rC}^{3}$ \\
\hline Staph. epidermidis & 11 & 3 & 9 & 24 & 10 & 18 & 16 & 8 & 11 & 12 & 1 & 9 & 9 & 2 & 7 & 4 & 2 & 4 \\
\hline Staph. aureus & 2 & 1 & 2 & 1 & & & 3 & 3 & 3 & 2 & & 2 & 3 & 2 & 2 & 2 & & 2 \\
\hline Group D. Strep & 2 & 2 & 2 & 1 & & 1 & 2 & 1 & 1 & 5 & 2 & 4 & 7 & 4 & 6 & 5 & 2 & 4 \\
\hline E. coli & & & & 3 & & 1 & 5 & 1 & & 3 & & & 6 & & 3 & 6 & & 1 \\
\hline Enterobacter & & & & 3 & & & 3 & & & 2 & & & & & & 1 & & \\
\hline Proteus & & & & & & & 2 & 1 & 2 & 1 & & 1 & 1 & & 1 & 1 & & 1 \\
\hline Klebsiella & & & & 2 & & & 5 & 1 & 1 & & & & 7 & & 1 & 9 & & 2 \\
\hline Citrobacter & & & & & & & & & & & & & 1 & & & & & \\
\hline Acinetobacter & & & & & & & & & & & & & 1 & & & & & \\
\hline Group B. Strep & & & & & & & & & & & & & 1 & 1 & 1 & & & \\
\hline Serratia & & & & & & & & & & & & & & & & 1 & & 1 \\
\hline
\end{tabular}

\footnotetext{
${ }^{1} N=$ number of stools with predominant organism.

${ }^{2} \mathrm{rG}=$ number of stools with organism resistant to gentamicin.

${ }^{3} \mathrm{rC}=$ number of stools with organism resistant to collistin.
} 


\section{DISCUSSION}

Studies about the use of oral aminoglycosides for the prevention of neonatal NEC have been presented in five previous reports. Of these, three studies $(6,8,12)$ have shown a statistically significant advantage from oral aminoglycoside prophylaxis, whereas two indicated no such difference $(5,16)$. The present study attempted to evaluate the bacteriologic effects of gentamicin compared with an alternative regimen. Colistin was selected as the alternative regimen because it is known to reduce Gram-negative colonization in the bowel, is not absorbed from the normal adult gut, and seems not to share cross-resistance with the aminoglycosides (10). Based on the results of the previous prophylaxis studies, and the Guidelines for the Ethical Conduct of Studies to Evaluate Drugs in Pediatric Populations from the American Academy of Pediatrics Committee on Drugs (1), a concurrent placebo group from the same patient population could not be included.

Following birth, the intestine becomes populated with enterococci and coliform organisms. Escherichia coli, Klebsiella spp., Enterobacter spp., and Proteus spp. are the most frequently isolated aerobic Gram-negative bacilli from newborns. It has been reported that $77-86 \%$ of $4-10$-day-old infants are colonized with aerobic Gram-negative bacilli regardless of gestational age (14). Furthermore, group D streptococci are found in $82 \%$ of 4-6-day-old infants, with a majority of these organisms being enterococci (14). After feeding is instituted, the Gram-negative bacteria of the feces increases markedly over a 3-day period. The number of organisms in the duodenum of infants $\left(10^{3}-10^{6}\right.$ per $\mathrm{ml}$ of intestinal contents) may be a thousand times as many as found in the adult (7). As the intestinal contents moves to the colon, density of organisms reach $10^{10}$ per $\mathrm{g}$ of stool (7). Abnormally increased proliferation of intestinal flora can result from decreased peristalsis, as is commonly seen in the small and/or sick premature baby.

The results of stool flora analysis in the present study showed a similar pattern of aerobic colonization to that previously reported. There was slightly greater suppression of bacterial growth by gentamicin than colistin particularly for $E$. coli and Klebsiella. Bell, et al. (3) reported that increased identification of these bacteria as dominant organisms in the gastrointestinal tracts of unaffected infants in a nursery occurred simultaneously with an increased attack rate of NEC (3). Furthermore, these investigators reported that gastric and fecal cultures during antibiotic therapy with oral gentamicin for NEC showed significantly decreased Gram-negative aerobic organisms (4). As in our study, they also indicated that Staph. epidermidis was the most frequent Grampositive aerobic organism in their isolates. This finding is probably due to the antibiotic-induced reduction of other species in the intestine. The \% of all predominant fecal organisms resistant to gentamicin was higher in the gentamicin treatment group than in the colistin treatment group; however, the ratio of gentamicin resistance to gentamicin sensitivity did not increase over the course of the 3-wk treatment period. Therefore, it is not certain that this difference between the treatment groups can be attributed to the administration of the oral antibiotic.

Resistance of $E$. coli and Klebsiella to colistin in our study groups was shown to be higher for these organisms when compared with gentamicin (17\% versus $9 \%$ at the end of $3 \mathrm{wk}$ ). The difference, although not significant, might be expected on the basis of a narrower antibiotic spectrum for colistin. Boyle et al. (5) reported an incidence of $47 \%$ kanamycin-resistant Gram-negative organisms in the stools of a group of infants who received NEC prophylaxis with kanamycin starting during the first wk of life and lasting 24 days. This compared with $24 \%$ resistance in stools of a control group during the same time period. There are several potential reasons why our resistance rates to the prophylactic antibiotics were lower than Boyle's. First of all, kanamycin was being used as a parenteral antibiotic in their nursery at the same time that oral kanamycin prophylaxis was ongoing. Second, the $\%$ of babies in their total nursery population receiving prophylaxis may have been greater than our $14 \%$ rate. Third, our nursery population consists only of babies born at the same hospital, whereas Boyle's group may have included transported babies. Finally, our study patients were started on oral antibiotics within $12 \mathrm{~h}$ of birth; Boyle's group were begun on medication at various times during the first and second wk of life.

Of the 100 patients in our study population, $38 \%$ received parenteral antibiotics at some time during their 3-week oral antibiotic course. There was no significant difference in this use between colistin and gentamicin groups. Previous reports have indicated that parenteral and oral antibiotics used together have an additive effect to suppress colonization (5). Furthermore, it has been reported that parenteral antibiotic usage in an intensive care nursery can be related to subsequent resistance to that antibiotic $(2,9)$. During our 11 -month study period, sensitivity to gentamicin of $E$. coli and Klebsiella from all infant culture sources in the nursery did not decrease.

The concerns raised about the possible emergence of resistant organisms after the use of oral NEC prophylaxis imply potential risk for individual patients as well as possible adverse changes in nursery residential flora. Our observations do not support these worries. Theoretically, colistin may be an acceptable prophylactic alternative if aminoglycoside resistance increases in the nursery; however, comparison of colistin with placebo must be done in future study. The use of any antibiotics for the prevention of necrotizing enterocolitis must be concurrently accompanied by surveillance of neonatal infection, nursery colonization and antibiotic sensitivity patterns to insure optimal efficiency.

\section{REFERENCES AND NOTES}

1. American Academy of Pediatrics Committee on Drugs: Guidelines for the ethica conduct of studies to evaluate drugs in pediatric populations. Pediatrics, 60:91 (1977).

2. Baker, C. M., Barrett, F. F., and Clark, D. J.: Incidence of kanamycin resistance among Eschericia coli isolates from neonates. J. Pediatr., 84: 126 (1974)

3. Bell, M. J., Shackelford, P., Feigin, R. D., Ternberg, J. L., and Brotherton, T. Epidemiologic and bacterial evaluation of neonatal necrotizing enterocolitis. $J$ Ped. Surg., 14: 1 (1979).

4. Bell, M. J., Shackelford, P. G., Feigin, R. D., Ternberg, J. L., and Brotherton, T. Alterations in gastrointestinal microflora during antimicrobial therapy for necrotizing enterocolitis. Pediatrics, 63: 425 (1979).

5. Boyle, R., Nelson, J. S. Stonestreet, B., Peter, G., Oh, W. Alterations in stool flora resulting from oral kanamycin prophylaxis of necrotizing enterocolitis. $J$. Pediatr., 93: 857 (1978).

6. Brantley, V., Hiatt, I., and Hegyi, T.: Effectiveness of oral gentamicin in reducing the incidence of necrotizing enterocolitis in treated and control infants. Ped Res. Abstract, 14: 4, 1001, 1980.

7. Brown, E. G., Ainbender, E., Henley, W. L., and Hodes, H. L.: Etiologic role of bacteria and intestinal function. In: Brown, E. G and Sweet, A. Y. Neonatal Necrotizing Enterocolitis, pp. 69-100 (Grune and Stratton, New York, 1980).

8. Egan, E. A., Mantilla, G., Nelson, R. N., and Eitzman, D. V.: A prospective controlled trial of oral kanamycin in the prevention of neonatal necrotizing enterocolitis. J. Pediatr., 89: 467 (1976).

9. Franco, J. A.; Eitzman, D. V., and Baer, H.: Antibiotic usage and microbial resistance in an intensive care nursery. Am. J. Dis. Child., 126: 318 (1973).

10. Goodman, L. S. and Gilman, A.: The Pharmacological Basis of Therapeutics (5th ed.) p. 1233, (Macmillan, New York, 1975).

11. Grylack, L. J.: Prevention of neonatal necrotizing enterocolitis. Perinatal Press, 3: 83 (1979).

12. Grylack, L. J. and Scanlon, J. W.: Oral gentamicin therapy in the prevention of neonatal necrotizing enterocolitis. Am. J. Dis. Child., 132: 1192 (1978).

13. Herbst, J. J. and Book, L. S.: Clinical characteristics. In: Brown, E. G. and Sweet, A. Y. Neonatal Necrotizing Enterocolitis pp. 24-40 (Grune and Stratton, New York, 1980).

14. Long, S. S. and Swenson, R. M.: Development of anaerobic fecal flora in healthy newborn infants. J. Pediatr., 91: 298 (1977).

15. McCracken, G. H. and Eitzman, D. V.: Necrotizing enterocolitis. Am. J. Dis. Child., 132: 1167 (1978).

16. Rowley, M. P. and Dahlenburg, G. W.: Gentamicin in prophylaxis of neonatal necrotizing enterocolitis. Lancet, 2: 532 (1978).

17. Standardized disc susceptibility test. Federal Register, 38: 2576 (1973).

18. Presented in part to the Society for Pediatric Research, San Antonio, TX, May, 1980

19. Requests for reprints should be addressed to: Dr. Lawrence Grylack, Division of Neonatology, Columbia Hospital for Women, 2425 "L" St., N.W., Washington, D.C. 20037

20. Received for publication July 6,1981 .

21. Accepted for publication December 7, 1981. 